\title{
Handwashing practice among healthcare providers in a teaching hospital in southern Nigeria
}

\author{
Bello Segun', Effa Emmanuel E², Okokon Enembe $\mathrm{O}^{1}$, Oduwole Olabisi $\mathrm{A}^{3}$ \\ 1. Department of Community Medicine, University of Calabar Teaching Hospital, Calabar, Nigeria \\ 2. Department of Internal Medicine, University of Calabar Teaching Hospital, Calabar, Nigeria \\ 3. Institute of Tropical Diseases Research and Prevention, University of Calabar Teaching Hospital, Calabar, Nigeria
}

doi: 10.3396/IJIC.v9i4.031.13

\begin{abstract}
Handwashing is the simplest and most cost-effective intervention to reduce transmission of nosocomial infections. In a cross-sectional descriptive survey, we investigated the practice, knowledge beliefs/attitude and determinants of handwashing among healthcare providers in a teaching hospital in southern Nigeria. A structured, self-administered questionnaire with both open-ended and close-ended questions, was used to collect data from respondents. Wards and clinical meetings were used as clusters to recruit participants. We found that more than two-thirds of participants had good handwashing practice. Inadequate facilities hindered compliance with handwashing guidelines. Nurses had significantly higher handwash frequency per patient contact than other healthcare providers. Majority (78.5\%) of participants knew that hands should be washed for at least 15 seconds, for effective prevention of nosocomial infection. Most (96.3\%) respondents believed that handwashing reduces the opportunity of infection transfer from one patient to another. Nurses and respondents who believed that facilities were adequate to prevent infection transmission, were more likely to comply. Provision of adequate facilities could improve handwashing practice.
\end{abstract}

Keywords: Health personnel; Hand disinfcetion; Infection control and Standards

\section{Introduction}

Handwashing is the simplest and most cost-effective intervention to reduce transmission of hospitalacquired infections. ${ }^{1-4}$ Health care workers put themselves and their patients at risk when they fail to observe routine hygiene practices. To reduce the incidence of hospital-acquired infections, guidelines recommend vigorous handwashing before and after every patient contact, or before and after contact with infected parts of the body. ${ }^{5}$

\section{Background}

Evidence-based guidelines for healthcare workers' hand hygiene exist. ${ }^{6}$ However, compliance is generally

\section{Corresponding author}

Dr. Segun Bello

Department of Community Medicine, University of Calabar Teaching Hospital, Calabar, Nigeria;

P.O.Box 1750, Calabar, Nigeria.

Email: drsegunbello@yahoo.com 
poor, usually less than average in most studies. ${ }^{7-9}$ It has been reported that healthcare providers with the greatest need (i.e. those who have high patient load) were less likely to comply. ${ }^{3}$ Handwashing practice may also vary among professional groups. ${ }^{1,4,5,10}$

Handwashing practice may be influenced by an interplay of knowledge, belief, attitude and socioeconomic factors. ${ }^{2}$ Knowledge about the benefits of handwashing was reported to significantly influence compliance with handwashing guidelines. ${ }^{11-14}$ Some authors belief that the poor compliance is purely attitudinal, and that knowledge may play little or no role. ${ }^{4,7}$ However, most relevant studies agree that type and availability of facilities have a significant effect on handwashing practice..$^{1-3,7}$

Hospital-acquired infection rates are highest in teaching hospitals. ${ }^{1}$ A single severe nosocomial infection may cost the hospital enormous resources per survivor, sometimes more than the entire annual budget for antiseptic agents used for hand hygiene., ${ }^{2,3}$ The implication of this is particularly pertinent for resource-poor countries like Nigeria.

While many studies have investigated a variety of interventionsto improvecompliancewith handwashing, the results have been equivocal, and long term continued compliance has been disappointing. ${ }^{3}$ Very few studies have investigated handwashing practice among healthcare providers in Nigeria. We are not aware of any study that has assessed the knowledge and determinants of handwashing practice among healthcare providers in Nigeria. We therefore, aimed to investigate the practice, knowledge, beliefs/attitudes and determinants of handwashing among healthcare providers in University of Calabar Teaching Hospital (UCTH), Nigeria. It is expected that information from this study would inform policy decisions on the control of hospital-acquired infections.

\section{Methods}

This was a cross-sectional descriptive study, carried out among health care providers at the University of Calabar Teaching Hospital. Doctors, nurses, community health extension workers, laboratory scientists and ward/theatre assistants were included in the study.
Cluster sampling technique was used to recruit respondents. Each ward in the hospital was visited once at a particular shift and questionnaires were distributed to the nurses and ward assistants on duty at that particular shift. Clinical meetings were used as clusters to reach doctors, while each laboratory was also visited at one shift. Potential respondents were given questionnaires to be returned through a designated colleague. We estimated a sample size of 356 for the study using a proportion of $63.3 \%$ (obtained from a previous study) ${ }^{10}$ in the Leslie-Kish formula for single proportion. ${ }^{15}$ There were a total of 1611 health care providers in the hospital.

A structured, self-administered questionnaire with both open-ended and close-ended questions, was used to collect data. The questionnaire had four sections: section one was on the personal data and work history of each respondent; section two assessed the handwashing practice among respondents and the facilities available for handwashing in the hospital; section three assessed the knowledge, attitude and beliefs of respondents on handwashing; and section four examined the factors influencing handwashing practice in the hospital.

Data collection was carried out over three months (December, 2009 through February, 2010). Data was managed with SPSS Statistics 14.0 (IBM Corp. NY, United States). Results were presented mainly as proportions. We presented the proportion with good handwashing practice with its $95 \%$ confidence interval. Mean frequency of hand wash per patient contact was calculated from the reported estimate of total hand wash per shift/day divided by the reported estimate of total patient contact per shift/day. Chisquare test was used to test associations between categorical variables. The level of significance was set at $p \leq 0.05$.

Ethical approval was sought and obtained for the study from the joint ethics review committee of the University of Calabar Teaching Hospital and the University of Calabar. Written informed consent was obtained from participants and confidentiality was ensured throughout the study. 


\section{Results}

Altogether, 400 questionnaires were distributed and 330 were retrieved giving a response rate of $82.5 \%$.

\section{Socio-demographic characteristics}

Mean age of respondents was $30.4 \pm 13.7$ years. One hundred and fifty (45.5\%) were nurses, $112(33.9 \%)$ were doctors, $22(6.7 \%)$ were ward assistants, 46 $(13.9 \%)$ were laboratory scientists and community health extension workers. About two-thirds (65.2\%) of respondents were female, most (93.9\%) had tertiary education while almost all respondents (99.4\%) were Christians. About half $(51.5 \%)$ of participants had worked for less than five years at the hospital (Table I).

\section{Handwashing practice}

Mean number of patient contact per shift among nurses was $15.4 \pm 10.6$; among ward assistants was $11.4 \pm 4.6$; while for doctors, mean number of patient

\begin{tabular}{|c|c|c|}
\hline Variable & $\begin{array}{r}\text { Frequency } \\
(\mathrm{N}=330)\end{array}$ & Percentage \\
\hline \multicolumn{3}{|l|}{ Age } \\
\hline $20-29$ & 73 & 22.0 \\
\hline $30-39$ & 172 & 52.1 \\
\hline $40-49$ & 75 & 22.7 \\
\hline$\geq 50$ & 10 & 3.1 \\
\hline \multicolumn{3}{|l|}{ Gender } \\
\hline Male & 111 & 33.6 \\
\hline Female & 219 & 66.4 \\
\hline \multicolumn{3}{|l|}{ Education } \\
\hline Primary & 6 & 1.8 \\
\hline Secondary & 14 & 4.2 \\
\hline Tertiary & 310 & 93.9 \\
\hline \multicolumn{3}{|l|}{ Professional group } \\
\hline Ward assistant & 22 & 6.7 \\
\hline Nurse & 150 & 45.5 \\
\hline Doctor & 112 & 33.9 \\
\hline Others & 46 & 13.9 \\
\hline \multicolumn{3}{|c|}{ Length of service (years) } \\
\hline$<5$ & 177 & 53.8 \\
\hline $5-10$ & 67 & 20.3 \\
\hline $11-20$ & 47 & 14.2 \\
\hline$>20$ & 39 & 11.7 \\
\hline
\end{tabular}

contact per day was $7.9 \pm 5.3$; and among the other professions (laboratory scientists and community health extension workers) it was $15.2 \pm 12.2$.

Mean frequency of handwash per patient contact among nurses was $1.13 \pm 0.97$; among ward assistant was $0.72 \pm 0.57$; among doctors was $0.78 \pm 0.45$, and among others (laboratory scientists and community health extension workers) it was $0.60 \pm 0.30$. Nurses had a significantly higher mean frequency of handwash per patient contact, than the other professionals $(\mathrm{F}=$ 8.996, $\mathrm{p}<0.001)$.

Majority (70.3\%: 95\% Cl $=65.4-75.2)$ of respondents had good handwashing practice, washing hands before and after every patient contact. About a fifth (22.6\%) of compliant respondents washed hands for less than 15 seconds, $46.9 \%$ washed hands for $15-30$ seconds while $30.5 \%$ washed hands for more than 30 seconds. About half $(51.9 \%)$ used water and ordinary soap, $44.1 \%$ used antiseptic solution, $2.8 \%$ used only water and $1.6 \%$ used spirit and swab most of the time. More than half (52.5\%) believed that the materials used for handwashing were inadequate to protect them and the patients from contracting infections.

\section{Knowledge, attitudes and beliefs}

Only half (50.3\%) of respondents knew that the most common route of transfer of hospital acquired infection was through contact between patients and healthcare providers, $19.7 \%$ said it was through used needles, $22.2 \%$ said it was through contacts between patients, $5.2 \%$ stated other routes, while $2.5 \%$ admitted they did not know. The majority (78.5\%) of the participants knew that hands should be washed for at least 15 seconds for effective prevention of nosocomial infection. Most (96.3\%) respondents believed that handwashing reduces the chance of infection transfer from one patient to another. More than a third $(35.3 \%)$ did not believe handwashing reduces the cost of medicines per patient. Most (95.3\%) believed handwashing should take place before and after every patient contact.

\section{Determinants of handwashing practice}

Three quarters $(75.5 \%)$ of respondents stated that facilities for handwashing were inadequate. Reasons for inadequacy included: inadequate water (20.9\%), 


\begin{tabular}{|c|c|c|c|c|}
\hline \multirow[t]{2}{*}{ Factor } & \multicolumn{2}{|c|}{ Handwashing Practice } & \multirow[t]{2}{*}{ Total } & \multirow[t]{2}{*}{ P-value } \\
\hline & Good & Poor & & \\
\hline Profession & & & & $<0.001$ \\
\hline Ward assistant & $18(81.8)$ & $4(18.2)$ & $22(100.0)$ & \\
\hline Nurse & $142(94.4)$ & $8(5.6)$ & $150(100.0)$ & \\
\hline Doctor & $59(52.7)$ & $53(47.3)$ & $112(100.0)$ & \\
\hline Others & $26(55.6)$ & $20(44.4)$ & $46(100)$ & \\
\hline Belief facility adequate & & & & $<0.001$ \\
\hline Yes & $76(94.5)$ & $5(5.5)$ & $81(100.0)$ & \\
\hline No & $166(66.8)$ & $83(33.2)$ & $249(100.0)$ & \\
\hline
\end{tabular}

absence of detergent/soap (29.1\%), unavailability of wash basins/sinks (6.1\%), sink(s) leaking (8.5\%), sinks too far $(7.3 \%)$, water not running in sinks $(30.6 \%)$, lack of clean towels (43.0\%), quality of soap is poor (29.1\%), lack of hand lotion/lubricants (40.3\%), and other reasons $(15.5 \%)$ (Figure 1 ).
Majority (91.8\%) of respondents expressed willingness to practice handwashing after every patient contact if adequate facilities were in place. Reasons given by $8.2 \%$ of respondents for unwillingness to practice handwashing after every patient contact, even if adequate facilities were in place, included: lack of

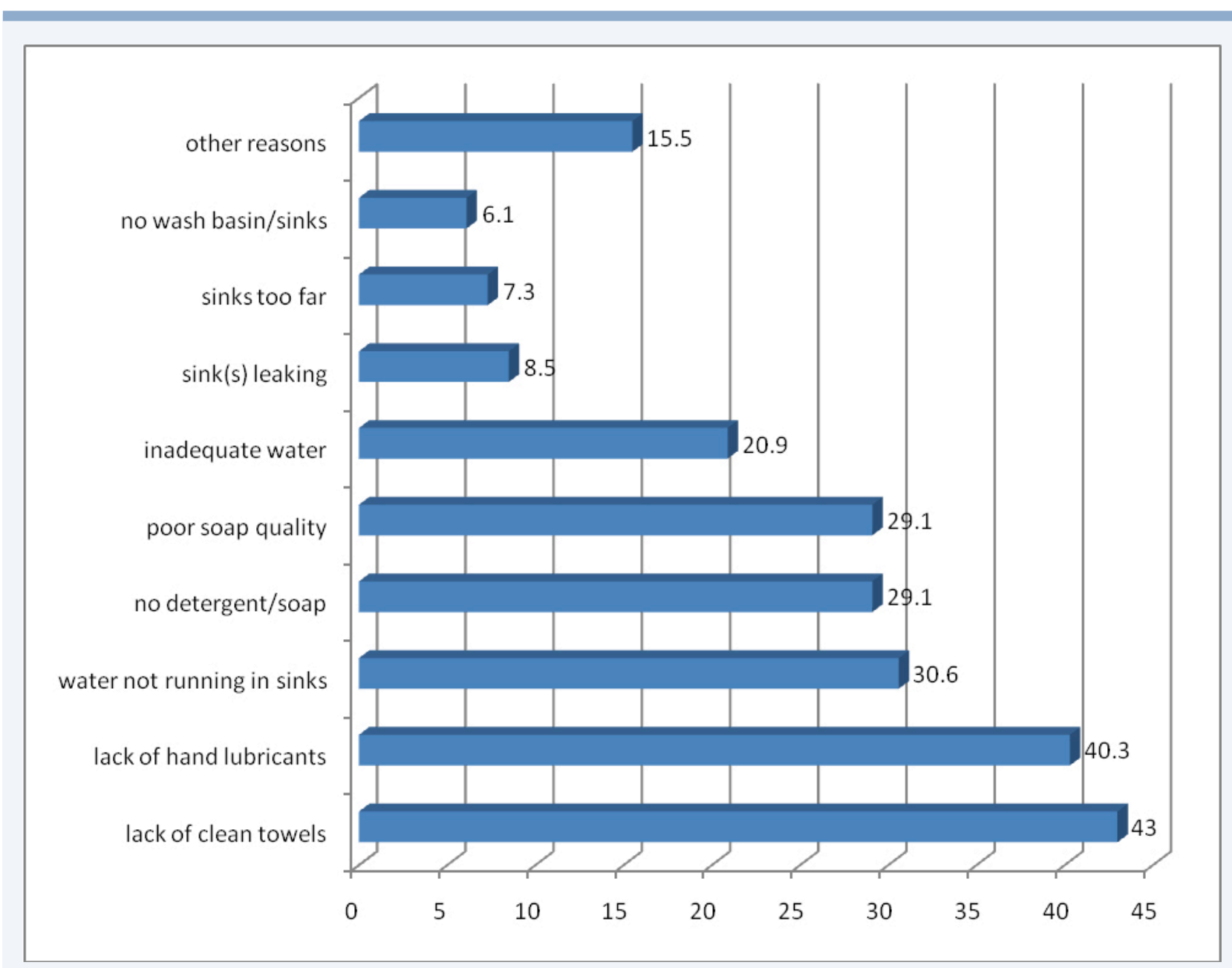

Figure 1. Reasons for inadequate facilities 


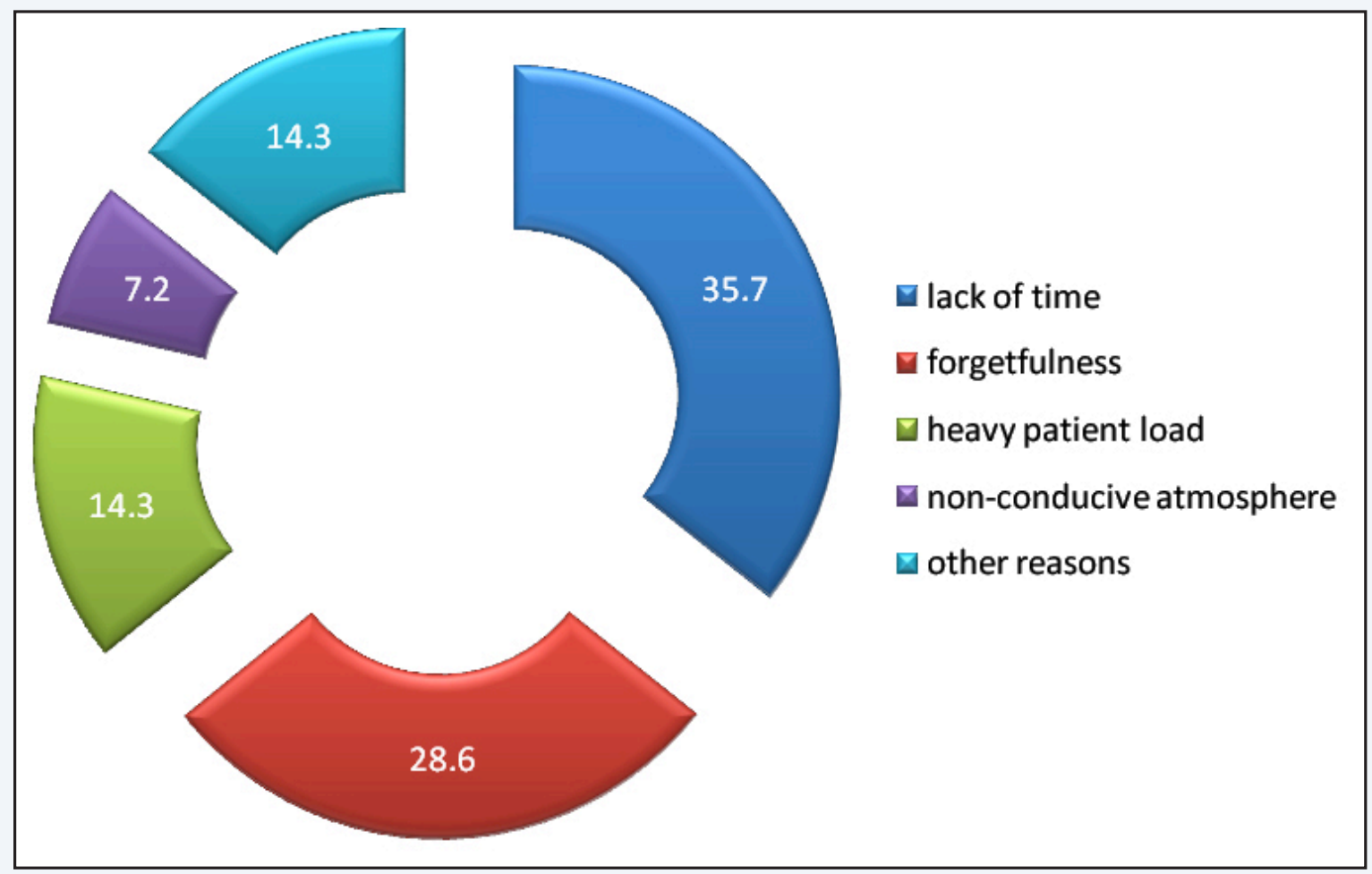

Figure 2. Reasons for unwillingness to practice handwashing guideline recommendations

time $(35.7 \%)$, heavy traffic of patients $(14.3 \%)$, nonconducive atmosphere (7.2\%), forgetfulness (28.6\%) and other reasons (14.3\%) (Figure 2).

More than two-thirds (67.9\%) were aware of infection control policy in the hospital. Acknowledged sources of infection control information among these respondents included posters $(32.0 \%)$, training $(52.7 \%)$, memo $(9.0 \%)$, and other sources $(6.3 \%)$.

Less than half $(41.8 \%)$ of respondents had attended training on hospital-acquired infections, infection control or handwashing practice in the last 10 years prior to survey. Less than half (39.4\%) were aware of the existence of infection control committee in the hospital. However, $96.4 \%$ expressed willingness to attend training on infection control.

Only two factors were significantly associated with handwashing practice in the hospital. Professional group of respondents was significantly associated with handwashing practice $\left(\mathrm{X}^{2}=63.85, \mathrm{p}<0.001\right)$; nurses were more likely to have good handwashing practice than the other groups of healthcare providers. Respondents who believed that facilities for handwashing were inadequate, were less likely to have good handwashing practice $\left(X^{2}=21.90, p<0.001\right)$ (Table II).

\section{Discussion}

Handwashing is an important practice in the control of nosocomial infections. Our study revealed that compliance with handwashing before and after patient contact was high. About $70 \%$ of respondents wash their hands before and after every patient contact while one-fifth of compliant respondents actually wash their hands for less than the recommended minimum time of 15 seconds. ${ }^{1}$ Compliance rate was above average in our study when compared to what was documented in some studies. ${ }^{4,16}$ Conversely, several other studies have reported below average compliance. ${ }^{1,7-9}$ Handwashing practice may be deemed satisfactory among healthcare providers in our study setting or respondent might have over-rated their personal compliance as had been previously reported. ${ }^{7}$ Differences in the level of compliance between studies may also be due to differences in study design, for example, Samuel et al. reported compliance level from a focus group discussion which may not be totally representative.

Less than half of compliant respondents used antiseptic solutions. More than $50 \%$ also believed 
that the materials they used for handwashing were not adequate to protect them and their patients against infections. Reports from another resource poor setting suggest a similar trend..$^{17}$ This has considerable implications. Though a good proportion of healthcare providers may wash their hands before and after every patient contact, the quality of the handwashing may not be adequate to prevent the transmission of some nosocomial infections.

We also found that mean number of handwashing per patient contact was significantly higher among nurses than among other healthcare professionals. Nursing training probably emphasizes hygiene in patient care. Studies have documented that some healthcare professional groups are worse than others in handwashing practice. ${ }^{1,4,5,16}$ Notably, a similar study among healthcare professionals in a neighbouring teaching hospital reported that doctors were more likely to wash their hands than nurses. ${ }^{18}$ This difference may be due to the fact that Alex-Hart and colleague did not restrict their study on handwashing to patient contact only.

About half of all respondents were not aware that the most common route of transmission of hospitalacquired infections was through contact between healthcare providers and patients, but majority were aware that each handwashing session should take place for at least 15 seconds. A similar study among healthcare providers in Kosova hospitals reported that $69 \%$ were aware that the most common route of hospital-acquired infections was through contact between healthcare providers and patients. ${ }^{11}$ Kennedy and colleagues reported a lower awareness rate (31\%) on recommended minimum time for handwashing. ${ }^{19}$ Knowledge could influence respondents' compliance with the handwashing guidelines. Healthcare providers also need to be well informed on available evidence which could be a potent motivator.

Most respondents in our study believed that handwashing should take place before and after every patient contact and most also believed that handwashing prevents transmission of infection from one patient to another. In a study conducted by Harris and coworkers, a similarly high proportion $(89 \%)$ of healthcare providers believed that handwashing was important in prevention of infection. ${ }^{7}$ It could be inferred that the attitude of healthcare providers towards handwashing practice was favourable and would be a fertile access for knowledge growth and behaviour change.

We found that compliance with handwashing guidelines has been hampered by inadequate facilities (water, soap, antiseptics, etc.) and heavy patient traffic among others, a situation also reflected in other studies. ${ }^{18}$ Compliance may be challenging in busy clinics where healthcare providers may need to attend to a large number of patients. This may call for adaptation of these guidelines; for example, total compliance may be enforced in high risk units where there is a higher risk of infection transfer, like the intensive care units. Other methods that are less timeconsuming e.g. the use of alcohol rubs, may be more effective in very busy clinics.

Only professional group and thebelief thathandwashing facility was adequate were significantly associated with compliance. Nurses were more likely to comply. Doctors have been reported to have least compliance in most studies. ${ }^{1,2,4,5,16}$ However, Alex-Hart reported that doctors were more likely to practice handwashing than nurses. ${ }^{18}$ Other studies have documented that handwashing compliance increases with the quantity and quality of facilities. ${ }^{1,2,3,7}$ Heavy workload, ${ }^{1,5,13,20}$ and lack of time ${ }^{1-3,12}$ reduce compliance.

Less than half have attended training on infection control in the last ten years and about the same proportion were not aware of the existence of an infection control committee in the hospital. However, most respondents expressed willingness to attend training on infection control.

A major limitation of our study is that we studied only self-reported handwashing practice among respondent and the perceived deterrents to compliance. A future study could incorporate direct observation of handwashing practice and an appraisal of the adequacy of facilities available for handwashing.

In conclusion, the practice of handwashing before and after contact with every patient was high among healthcare providers in our hospital. However, the 
quality of handwashing may not be adequate to prevent infection transmission. Regular in-service training on handwashing guidelines and infection control as well as awareness reinforcement in form of posters in strategic locations could improve compliance with handwashing practice. Provision of adequate and quality facilities, realistically adapted to the needs, may improve handwashing/handhygiene practice and nosocomial infection control. Establishing what interventions would likely produce the best outcome requires further research.

\section{Acknowledgement}

We thank the healthcare providers who spared their time to participate in this study.

\section{References}

1. Samuel R, Almedom AM, Hagos G, Albin S, Mutungi A. Promotion of handwashing as a measure of quality of care and prevention of hospital-acquired infections in Eritrea: the Keren study. African Health Sciences 2005; 5(1): 4-13.

2. Boyce JM. Antiseptic Technology: Access, Affordability and Acceptance. Emerg Infect Dis 2001; 7(2): 231-233. http:// dx.doi.org/10.3201/eid0702.010216

3. Lautenbach E. Practices to Improve Handwashing compliance. Agency for Healthcare Research and Quality, U.S Department of health and Human Services www.hhs.gov.

4. Rao MH. Knowledge, Attitude and Practice, Patterns of Handwashing in major public sector hospitals of Karachi. Park J Med Res 2006; 45(4): 87-92.

5. Pittet D, Simon A, Hugonnet S, Pessoa-Silva CL, Sauvan V, Perneger TV. Hand hygiene among physicians: performance, beliefs and perception. Annals of Internal Medicine 2004; 141(1): 1-38. http://dx.doi.org/10.7326/0003-4819-141-1200407060-00008

6. Creedon SA. Healthcare workers' hand decontamination practices: compliance with recommended guidelines. J Adv Nurs 2005; 51(3): 208-216. http://dx.doi.org/10.1111/j.13652648.2005.03490.x

7. Harris AD, Samore MH, Nafziger R, Dirosario K, Roghmann MC, Carmeli Y. A survey on handwashing practices and opinions of healthcare workers. The journal of hospital Infection 2000; 45(4): 319-321. http://dx.doi.org/10.1053/ jhin. 2000.0781
8. Patarakul K, Tau-Khum A, Kanha S, Padung-Pean D, Jaichaiyapum OO. Cross-sectional survey of hand hygiene compliance and attitudes of health care workers and visitors in the intensive care units of King Chulalongkorn Memorial Hospital. J Med Assoc Thai 2005; 88 Suppl 4: S287-293.

9. Mirzaeik AM, Mundy LM, McLaws ML. Assessment of knowledge, attitudes and practices regarding isolation precautions among Iranian healthcare workers. Infect Control Hosp Epidemiol 2005; 26(1): 105-108. http://dx.doi. org/10.1086/502495

10. Suchitra JB, Devi NL. Impact of education on knowledge, attitudes and practices among various categories of healthcare workers on nosocomial infections. India Journal of Medical Microbiology 2007; 25(3): 181-187. http://dx.doi. org/10.4103/0255-0857.34757

11. Raka L, Kalenic S, Zoutman D, et al. Knowledge, attitudes and practices of healthcare workers in Kosovo hospitals regarding nosocomial infections. European Society of Clinical Microbiology and Infectious Diseases. $16^{\text {th }}$ European Congress of Clinical Microbiology and Infectious Diseases Book of Abstracts. Page 659.

12. Barrett R, Randle J. Hand hygiene practices: nursing students' perceptions. J Clin Nurs 2008; 17(14): 1851-1857. http:// dx.doi.org/10.1111/j.1365-2702.2007.02215.x

13. Akyol AD. Hand hygiene among nurses in Turkey: opinions and practices. / Clin Nurs 2007; 16(3): 431-437. http://dx.doi. org/10.1111/j.1365-2702.2005.01543.x

14. Wisniewski MF, Kims S, Trick WE, Welbel SF, Weinstein RA, Chicago Antimicrobial Project. Effect of education on hand hygiene beliefs and practices: a 5-year program. Infect Control Hosp Epidemiol 2007; 28(1): 88-91. http://dx.doi. org/10.1086/510792

15. Kish L. Survey sampling. Wiley Interscience Publication. New York; 1965.

16. Suchitra JB, Devi NL. Impact of education on knowledge, attitudes and practices among various categories of healthcare workers on nosocomial infections. India Journal of Medical Microbiology 2007; 25(3): 181-187. http://dx.doi. org/10.4103/0255-0857.34757

17. Abd Elaziz KM, Bakr IM. Assessment of knowledge attitude and practice of hand washing among health care workers in Ain Shams University hospitals in Cairo. I Prev Med Hyg 2009; 50(1): 19-25.

18. Alex-Hart BA, Opara PI. Handwashing practices amongst health workers in a teaching hospital. American Journal of Infectious Diseases 2011; 7(1): 8-15. http://dx.doi. org/10.3844/ajidsp.2011.8.15

19. Kennedy AM, Elward AM, Fraser VJ. Survey of knowledge, beliefs and practices of neonatal intensive care unit healthcare workers regarding nosocomial infections, central venous catheter care and hand hygiene. Infect Control Hosp Epidemiol 2004; 25(9): 747-752. http://dx.doi.org/10.1086/502471

20. Cohen HA, Kitai E, Levy I, Ben-Amitai D. Handwashing patterns in two dermatology clinics. Dermatology 2002; 205(4): 358-361. http://dx.doi.org/10.1159/000066421 